\title{
DEUX MÉTHODES DE SÉLECTION \\ ET LEURS APPLICATIONS A L'AVICULTURE
}

PAR

J. P. BOYER

Station de Recherches Avicoles, Jouy-en-Josas

\section{PLAN DU MÉMOIRE}

\section{A. - Examen critique :}

I. - La sélection massale.

II. - La sélection génotypique.

III. - Conclusion.

\section{B. - Applications :}

I. - Premières études quantitatives.

II. - Organisation générale de la sélection.

III. - Organisation particulière de la sélection.

IV. - Conclusion.

\section{A. - EXAMEN CHITIQUE}

L'histoire de la sélection nous montre une évolution du simple au complexe, du subjectif à l'objectif mathématique. VIRGILE rapporte que les Romains choisissaient pour semences leurs grains les plus lourds. C'est donc sur l'apparence - nous disons aujourd'hui sur le phénotype - que les premiers essais de sélection ont été réalisés.

Mais la poule étant naturellement destinée à pondre des œufs et à fournir de la chair à l'homme, celui-ci chercha les moyens de reconnaître l'animal productif à certains signes extérieurs.

L'introduction du nid-trappe et de la comptabilité permit ensuite un progrès considérable de l'aviculture, allant de pair d'ailleurs avec le perfectionnement de techniques d'élevage telles que l'alimentation et l'habitat.

Mais l'outil génétique et statistique devait permettre une compréhension et un développement plus grand encore de la production, en ne jugeant plus l'animal sur lui-même, mais aussi sur son patrimoine héréditaire, garantie plus valable de l'excellence de ses enfants. 
Cette évolution n'est pas terminée. Si la sélection massale nous apparaît comme insuffisante, nous entrevoyons aussi les limites d'une sélection génotypique simple. Des notions contemporaines telles que l'héritabilité et 1'index de production apportent une lueur nouvelle sur ce que nous avons souvent tendance à considérer comme immuable.

\section{LA SELECTION MASSALE}

\section{Telle qu'elle est}

La majorité des ouvrages de vulgarisation présente la sélection massale sous le jour d'une sélection phénotypique. Dans cette méthode, l'individu est jugé sur son extérieur, son standard, sa conformation, sa production (généralement réduite au contrôle du nid-trappe), enfin sur des corrélations phénotypegénotype. Cette méthode est donc extrêmement simple ; malheureusement, telle qu'elle est, elle s'avère insuffisante du fait qu'elle ne tire aucun parti de notions relativement sommaires de génétique et de statistique.

Ainsi, le jugement de l'animal sur son phénotype n'est en fait que le jugement de l'interaction de son génotype avec le milieu. Dans le cas simple d'un gène dominant, nous savons que le phénotype de l'individu hétérozygote cache un gène récessif. La sélection massale ne sera donc efficace que dans le cas d'un caractère récessif monofactoriel ou d'un caractère polygénique à haute héritabilité, c'est-à-dire indépendant du milieu (ASMUNDSON et LERNER, I95I), alors que, dans le cas d'un caractère dominant, ou faiblement héritable, son efficience restera limitée.

I. - Ainsi, J. DRYDEN (I92I) a pu en dix ans augmenter la production de Leghorns blanches de 107 à 2 I 2 œufs et de Rocks barrées de 86 à 2 I 5 wufs. Mais encore peut-on noter que ce plafond était pratiquement atteint en six ans.

On connait les expériences de PEARL (IgII) qui diminua, contre son désir, la production de son troupeau en utilisant la sélection massale. Sans aller aussi loin, SuRFACE (IgOg) montra que les poules de 200 œufs ne donnent pas de filles meilleures que les poules de I50 à 200 œufs. Malgré les expériences de HaLL (r9.35) montrant que des coqs issus de poules différant par leur ponte de plus de $5^{\circ}$ cufs par an font apparaître des différences significatives dans leurs descendances, les cas de non-transmissibilité sont trop nombreux pour qu'on attende de la sélection massale plus que celle-ci ne peut donner (JULL, I934).

Nous dirons donc que la ponte est un caractère à faible héritabilité et, par suite, mauvaise matière à sélection massale.

II. - La sélection sur la conformation sera moins aléatoire, du fait de l'héritabilité intermédiaire de caractères tels que la longueur du bréchet ou le tour de poitrine (Asmundson, I95I). En fait, la littérature antérieure à la 
notion d'héritabilité soulignait déjà l'efficacité et la simplicité de la sélection pour la chair eu égard à la sélection pour la ponte (Ponsignon, I942 ; ColE et Bruckner, i94I ; Goodale, I935, I94I).

III. - - Le cas d'un caractère dominant est encore plus simple à étudier. Nous savons que l'hétérozygote $\mathrm{R} r$ à crête rosacée transmettra le gène $r$ à ses enfants. En éliminant de la reproduction les individus à crête simple, après $n$ générations de sélection, la diminution de la fréquence $q$ du gène $r$ devrait suivre la loi de JenNings (IgI3) :

$$
q_{n}=\frac{q_{0}}{\mathrm{I}+n q_{0}} .
$$

Cette progression est d'abord rapide, puis s'amenuise en quelques années. Si nous supposons (cas le plus favorable pour débuter une sélection) que $q_{0}=\mathrm{I} / 2$, après dix générations de sélection massale, nous avons encore $q_{1_{0}}=$ $\mathrm{I} / \mathrm{I} 2$, alors qu'en huit générations, nous avions $q_{8}=\mathrm{r} / \mathrm{I} 0$.

Le cas présent est compliqué du fait que, malgré la sélection, les individus $q^{2}$ réapparaissent avec un taux d'environ I I p. IOO L. P. CochEZ (I95I) a montré comment la sélection gamétique du gène $r$ compensait la sélection artificielle du caractère crête rosacée. La sélection massale est impuissante à passer cet. obstacle.

IV. - Notons également que l'infertilité liée à la crête rosacée (démontrée par la valeur sélective de l'individu $\mathrm{R} r$ supérieure à celle de l'individu $\mathrm{RR}$ ) est un argument contre la position a priori des standards de perfection.

Le plus grave est que l'aviculteur s'attache aux exigences du standard et, augmentant par là même le nombre de ses critères de sélection, diminue la pression de celle-ci quant aux caractères de production - d'autant plus inutilement que maintes exigences des standards n'ont aucun rapport avec la productivité (LERNER, I950).

V. - Quant aux corrélations phénotype-génotype, elles n'ont pas cessé d'être dénoncées (Marble, I932 ; Milleer et Carver, I934; JUlL, I933; LERNER, I950, BRYAND et STEPHENSON, 1945). Nous n'y reviendrons pas.

\section{Telle qu'elle pourrait être}

L'interaction génotype-milieu et la notion corollaire d'héritabilité nous ont expliqué les limites de la sélection massale, mais aussi ses possibilités dans le cas de caractères récessifs monofactoriels ou de caractères polygéniques hautement héritables.

La difficulté de sélectionner plusieurs caractères et la diminution de la pression de sélection (inverse du pourcentage d'individus choisis comme reproducteurs) des caractères économiques qui suit la sélection des caractères ra- 
ciaux, conduiront donc l'éleveur à ne pas tant rechercher les perfections du standard que les qualités de production - ou bien, à élever un troupeau dont les caractères phénotypiques seront récessifs.

I. - Sous ce rapport, la race Rhode Island Red offre un grand nombre de traits récessifs : crête simple $r r$, patte jaune ww, tarse non emplumé, $s_{1} s_{1} s_{2} s_{2}$, quatre doigts $p_{0} p_{0}$ plumage doré $s(s)$, non extension du noir $e e$, absence du blanc dominant $i$.

L'effort de sélection pourra porter par exemple sur l'emplumement rapide (récessif fixé au sexe $k(k)$ dont l'avantage est multiple : sexage à la naissance, en croisement avec des poules $\mathrm{K}$ ) ; protection contre le froid, d'où meilleure utilisation de la ration et croissance plus rapide (HuTro, I949, suggère la possibilité d'une action pléiotropique) ; enfin, le poulet d'engrais à I2 semaines est plus monnayable lorsqu'il est bien emplumé.

D'autre part, la sélection des caractères dominants est possible, mais l'homozygote dominant ne sera jamais complètement fixé par la sélection massale, sauf cas de disparition fortuite de l'allèle récessif.

II. - Restent les caractères polygéniques. Rapprochant l'intérêt pour la production de la chair du gène $k$ de l'héritabilité intermédiaire de caractères phénotypiques, tels que le tour de poitrine et la longueur du bréchet, et de la haute héritabilité du poids du corps et de la vitesse de croissance, nous pouvons prévoir que la sélection massale trouvera sa meilleure utilisation dans l'élevage orienté vers la production de la chair et non celle des œufs.

Sans doute, une méthode génotypique serait plus efficace, mais la simplicité de la sélection massale et la connaissance de ses possibilités permettent d'en tirer un très bon parti.

III. - Quant à la ponte, l'utilisation du nid-trappe ne doit pas se borner à un simple relevé d'œufs pondus. Le nid-trappe permet également de tester la précocité de la ponte, son intensité, la persistance, les pauses d'hiver, voire la couvaison. C'est en ces cinq facteurs indépendants que GOODALE (IgI8) a remarquablement décomposé la physiologie de la ponte. Cette analyse de la ponte et la sélection simultanée des composantes limitatives permettent un progrès supérieur à celui de la sélection successive (LERNER, I950).

D'autre part, la ponte pourra être étudiée sur les cufs : poids, forme, couleur et même résistance et transparence de la coquille.

Mais la faible héritabilité des caractères de la ponte ne permet pas à la sélection massale un progrès considérable (cf. J. DRYDEN, etc... cités plus haut).

On peut raisonnablement penser avec PÉTrov (I935) que les périgrinations de la sélection massale sont allées de pair avec l'amélioration des techniques, telles l'alimentation et l'habitat. C'est donc en grande partie la vulgarisation de ces techniques qui permettra au producteur d'œufs d'atteindre un plafond respectable d'un peu plus de 200 œufs, la sélection massale n'étant 
efficace que dans un troupeau peu ou pas amélioré (cf. Loi de JENNINGS, expériences de DRYDEN).

\section{II. - LA SELLCTION GENOTYPIQUE}

\section{Principes}

La génétique des populations n'a pas encore, à notre connaissance, donné le jour à une ouvre de synthèse réunissant les travaux de GaLTon, Hardy, Weinberg, Wright, Lush, Lerner, Mather... d'où son aspect quelque peu hermétique.

Mais son application à la sélection animale est un de ses aspects les plus séduisants.

On peut dire que toute sélection génotypique se ramène à l'étude de la ségrégation des gènes que permet l'analyse génétique et statistique. Or, qui dit étude de la ségrégation implique la nécessité de suivre une descendance bien déterminée. Les analyses sommaires de génétique formelle sont classiques, qui déterminent une partie du patrimoine héréditaire de la $\mathrm{F}_{\mathbf{0}}$ par l'analyse de la $F_{2}$. Mais l'étude de la $F_{1}$ peut être extrêmement utile, voire nécessaire. C'est cette étude qui constitue le progeny-testing de la $\mathrm{F}_{\mathbf{0}}$ - ou sib-test de la $\mathrm{F}_{\mathbf{1}}$. Dans ce cas, les géniteurs $\mathrm{F}_{0}$ seront choisis sur la valeur moyenne de leur descendance, les géniteurs $F_{1}$ sur leur écart à cette moyenne.

Mais il est clair que, dans le cas de caractères hautement héritables, la sélection massale sera supérieure à la sélection globale d'une famille, du fait que les individus les moins bons de cette famille transmettront à leurs enfants leur moindre valeur avec une forte probabilité. D'une façon plus frappante encore, on n'imagine guère de garder les crêtes simples d'une famille qui présente un fort pourcentage de crêtes rosacées.

Nous aurons donc à opérer une sélection combinée, individuelle et familiale, qui sera la plus efficace, ce qui revient à dire que les géniteurs seront également choisis sur leurs propres performances.

I. - Mais suivre une descendance implique la servitude de constituer des parquets de reproduction, où nous mettrons dix poules et un coq (puisque celui-ci ne laisse pas de témoin de sa paternité analogue à l'œuf de la poule). Les œufs de chaque poule devront être contrôlés au nid-trappe et marqués. L'éclosion s'effectuera en cases séparées pour chaque mère. Les poussins seront bagués et nous relèverons sur une même feuille le numéro de la mère et ceux de ses enfants.

Moyennant cette condition d'état-civil, nous pourrons mesurer tout ce que nous voudrons et faire toutes sortes de tests, en attribuant à tels parents la valeur moyenne de tels individus, d'où le choix des meilleurs parents et des meilleurs enfants des meilleures familles.

D'autre part, les caractères peu héritables permettront des tests de signi- 
fication d'autant meilleurs que la famille sera plus nombreuse et plus homogène. Il est évident que cela est impossible en sélection massale et que seule la sélection génotypique permettra d'accumuler des gènes favorables que l'action du milieu masque sur l'individu.

II. - Dans le cas de caractères monofactoriels dominants, le succès de la méthode génotypique sera complet. Ainsi, l'individu $\mathrm{R} r$ croisé avec $r r$ sera détecté par moitié de ses enfants $(r)$, alors que l'individu RR donnera toujours des enfants (R). La méthode est primaire, alors que la sélection massale est incapable d'en tirer parti. Pourtant, si la sélection génotypique permet des progrès certains et plus rapides que ceux de la sélection massale (par exemple, l'hétérozygote $\mathrm{R} r$ est décelé en une set1le génération ; le test des moyennes compense en quelque sorte la fluctuation), sous la forme que nous lui donnons, elle n'est pas parfaite.

Ainsi, dans l'exemple classique de la crête rosacée, nous aurons une certitude d'hétérozygotie, mais seulement une présomption d'homozygotie: $p=\mathrm{I}-(1 / 2)^{n}$.

III. - Dans le cas des caractères polygéniques, le problème sera beaucoup plus complexe. Les caractères à haute héritabilité seront bien perpétués par sélection massale (mieux encore par sélection familiale y combinée) - mais les caractères peu héritables seront encore entachés d'une erreur due au milieu.

Une première amélioration sera donc de ne pas élever séparément les familles, mais ensemble, de façon à réduire la fluctuation, et d'augmenter le nombre des descendants.

$$
\left(\lim h_{f u}^{2}=\frac{r_{g} h^{2}}{r_{g} h^{2}+\mathrm{C}^{2}} \quad \text { quand } n \rightarrow \infty\right)
$$

\section{Méthodes}

Mais une fois esquissés les principes et les servitudes de la sélection génotypique, il convient de faire une discrimination entre ses utilisations possibles.

I. - Nous pourrons nous contenter de choisir les meilleures familles; nous avons déjà émis une réserve précédemment. Nous pourrons choisir entre la sélection d'un caractère, puis d'un autre, ce qui peut créer des corrélations fâcheuses en cours de sélection. (On cite des souches de Leghorns sensibles à l'avitaminose $\mathrm{B}_{\mathbf{1}} ; c f$. plus haut l'infertilité liée au gène $\mathrm{R}$ ) et la sélection simu1tanée de plusieurs caractères (mais ceci aux dépens de la pression de sélection).

Enfin, la connaissance du pourcentage d'héritabilité devrait permettre, tout au moins dans l'avenir, la construction d'index de production qui, en rendant compte objectivement de la valeur utilitaire d'un individu, se substitueraient avec avantage aux " échelles de points " arbitrairement dressées. Ainsi, nous pourrons décomposer un caractère $\mathrm{X}$ entre deux éléments (par 
exemple, A pour le poids et B pour la couleur de l'œuf) affectés d'un coefficient économique : $\mathrm{X}=m \mathrm{~A}+n \mathrm{~B} . \mathrm{X}$ pourra à son tour rentrer dans la formule d'un index de production, tenant compte à la fois de son importance économique et de son taux d'héritabilité.

Par considération des gains probables, LERNER (I950) a montré par le calcul que la sélection fondée sur un index de production est plus efficiente que la sélection simultanée de plusieurs caractères, elle-même supérieure à la sélection successive.

II. - D'autre part, nous aurons également à choisir un système d'élevage. Ainsi, l'inbreeding augmentera la corrélation génétique entre individus et par suite, 1'homogénéité, mais chacun sait les dangers de la consanguinité aveugle. Le croisement permettra l'analyse génétique des caractères phénotypiques ou l'étude des phénomènes d'hétérosis, mais étalera les effets de la ségrégation. La panmixie pourra être appliquée en sélection massale, mais celle-ci est impuissante à passer certains obstacles. Enfin, chaque système a ses avantages et ses inconvénients. L'éleveur " génotypiste " qui voudra augmenter son effectif pourra utiliser deux coqs pour un même parquet "double shift ", évitant ainsi l'c inbreeding ", mais aux dépens de la pression de sélection. L'effet inverse se produira en utilisant un seul coq.

Le système d'élevage utilisé dépendra donc du but poursuivi et pourra varier selon les besoins. D'une façon très générale, nous pourrons choisir entre une consanguinité assez souple et une consanguinité étroite suivie de croisements (technique utilisée aux U.S.A. pour la production de poulets hybrides et depuis longtemps classique en génétique végétale).

Nous pouvons résumer ainsi la difficulté de préciser une marche à suivre :

L'inbreeding est la méthode la plus efficace de fixation des caractères. Mais les caractères économiques sont détériorés par l'“ inbreeding » et la fixation des caractères économiques désirables est, par suite, peu vraisemblable.

La méthode à appliquer se ramènera donc à une question d'objectifs.

\section{III. - CONCLUSIION}

'Tout est loin d'être dit. Ainsi, nous ne nous sommes pas arrêtés sur la détermination des caractères, la notion d'héritabilité, les phénomènes d' " overdominance ", toutes questions qui auraient alourdi ces quelques pages. Nous pouvons dire cependant que si la sélection massale est, à de multiples points de vue, inférieure à la sélection génotypique, elle a cependant des mérites non négligeables.

D'autre part, la sélection génotypique offre plusieurs aspects sinon de ses principes, du moins de ses méthodes. Nous ne commettrons donc pas la profonde erreur de penser qu'il suffit de "faire de la sélection génotypique " pour doubler sa production. 
Nous avons plutôt le dessein de réhabiliter une méthode simple et d'esquisser la complexité de l'analyse, dans un but de vulgarisation possible :

- afin d'éviter les abus qui s'attachent à la qualité de "sélectionneur ";

- afin d'apporter aux aviculteurs le profit qu'ils peuvent tirer de la sélection massale combinée au perfectionnement des techniques d'élevage et de réserver à ceux qui ont déjà atteint le plafond de ce système, ou qui en ont la compréhension critique, la possibilité d'aborder une méthode plus objective.

Mais nous avons le désir d'aller plus avant et de rendre plus efficientes encore les données de l'hérédité.

\section{B. - IPPLICATIONS}

Les objectifs postulés au départ par la Station de Recherches Avicoles en matière de sélection furent le contrôle des aptitudes économiques de quelques races françaises et l'orientation génotypique de la sélection. Nous séparerons en deux périodes l'activité de notre Service: les premières études quantitatives d'une part; d'autre part, l'organisation et la réalisation de la sélection génotypique.

Il est évident, en effet, que la nécessité de constituer un troupeau ne nous permettrait pas, dès la première année, d'éprouver une descendance - mais du moins d'enregistrer des informations sur la productivité de quelques races nationales, puis de constituer des parquets de reproduction par sélection massale.

C'est donc à compter du I er janvier 1952 que le programme de sélection génotypique est entré en vigueur.

\section{I. - PREMIERES ETUDES QUANTITATIVES}

Dans le but de dresser l'inventaire des aptitudes utilitaires moyennes de races françaises, le troupeau de Jouy fut constitué en I95 I par quinze souches réparties en cinq races. L'une, la Light-Sussex, race étrangère améliorée, devait servir de terme de comparaison. Les autres étaient les plus représentatives de la variété de nos races: Faverolles, race lourde à grande vitesse de croissance ; Gâtinaise, bonne pondeuse ; Marans argentée, pour la qualité de l'œuf ; Bresse blanche, race légère à chair fine.

Chaque race était représentée par trois souches, fournies sous forme d'œufs à couver, venant de trois élevages choisis pour leur réputation et de situations géographiques aussi différentes que possible.

Nos premières études ont porté sur l'incubation, la croissance, la mortalité et la ponte d'automne.

Chacune de ces études s'est subdivisée en différents relevés, puis en tests entre souches et entre races.

Ainsi, nous avons étudié l'incubation en la décomposant en œufs reçus, 
incubés, féconds, en germes morts aux $5^{\mathrm{e}}$ et $18^{\mathrm{e}}$ jours, en poussins morts en coquille, éclos, nés viables, et exprimé le pourcentage d'éclosion par rapport aux cufs reçus, incubés et féconds. Ensuite, nous avons testé les différences de pourcentage de chaque catégorie entre souches et entre races.

De même, l'étude de la croissance a porté sur le poids de l'œuf, les poids à la naissance, à huit jours, un mois, six et huit semaines et à trois mois.

L'étude de la mortalité allait de la naissance à deux mois.

Enfin, la ponte fut contrôlée au nid-trappe et les œufs observés durant le mois de décembre quant au poids, à la forme, à la couleur et à l'apparence de la coquille.

Sans nous arrêter sur des chiffres, qui allongeraient considérablement cet exposé, disons les conclusions générales auxquelles nous sommes arrivés:

I. - La Light-Sussex ne s'est pas avérée un témoin bien utile, dans la mesure où nous attendions d'elle qu'elle nous montre une valeur vers laquelle tendre nos efforts. En effet, ses résultats d'incubation étaient nettement inférieurs à ceux de la Bresse (environ $7 \mathrm{I}$ p. Ioo de nés viables/œeufs incubés, contre $85 \mathrm{p}$. IOO), sa croissance inférieure à celle de la Faverolles (760 g à huit semaines contre $870 \mathrm{~g}$ ), sa mortalité supérieure à celle de toutes les autres races, sa ponte légèrement supérieure à celle de la Gâtinaise.

Nous avons donc décidé de réduire l'effectif Sussex et de ne plus considérer cette race que comme un témoin.

II. - Les résultats de toutes les mesures ont montré une hétérogénéité très grande au sein d'une même race comme au sein d'une même souche.

Ainsi, le poids moyen à huit semaines des trois souches de Gâtinaises a lait de 690 à $800 \mathrm{~g}$; la mortalité des Bresse à deux mois, allait de 5 à I I p. Ioo.

De même, le poids des œufs à l'intérieur d'une même souche de Marans allait de 50 à $7 \mathrm{I}$ gr et la couleur a dû être étalonnée d'après quatre gammes différentes (unis, granulés, pigaillés, mauves). La ponte d'une souche de Faverolles allait de zéro à 50 œufs en quatre mois.

Malgré ces différences considérables, le test de STUDENT-FISHER n'apportait pas toujours de conclusion significative, soit parce que l'effectif était trop restreint, soit parce que la variance des distributions était trop élevée.

III. - I'une façon générale, les performances enregistrées ont été médiocres, particulièrement en ce qui concerne la ponte (le meilleur sujet, une Gâtinaise, a pondu 85 oufs en quatre mois; la moyenne du troupeau n'atteignait pas 40 œufs). La nécessité de constituer nos parquets de reproducteurs par sélection massale et la modestie des locaux disponibles nous ont cependant permis d'effectuer un choix assez sérieux, du fait de l'étalement des distributions. Mais la médiocrité des moyennes et l'élimination des porteurs de germes de la pullorose ne nous ont pas garanti un tri très promet- 
teur. Aussi, nous avons été obligés de réduire l'effectif Faverolles à un seul parquet, dont la moyenne de ponte n'excédait pas 3I œufs.

Les autres races, Gâtinaise, Marans et Bresse, ont été maintenues en souches "pures ", parce que leur variabilité laissait à penser que ces souches n'étaient précisément pas pures, de façon à pouvoir les suivre à nouveau en I952 et à se réserver des possibilités de non-consanguinité par la suite.

Restreints à douze parquets de reproducteurs, nous avons cherché à avoir en 1952 le plus grand nombre de descendants et le moindre inbreeding possible. Nous avons donc échelonné la période d'incubation sur neuf semaines, comprenant deux séries d'incubations réservées à un coq chacune.

Ainsi, pour tel parquet, les enfants du $\operatorname{coq} A$ et de la poule $n^{\circ}$ I ne seront pas parents des enfants $d u \operatorname{coq} B$ et de la poule $n^{\circ} 2$, ce qui permettra donc en 1953 de dédoubler nos souches ; étant postulé au départ que nos reproducteurs I95 I n'étaient pas parents (ceci, étant donnée la variabilité des résultats et l'ignorance totale des antécédents de nos souches).

Nos reproducteurs ont été choisis, quand la valeur des performances permettait plusieurs critères, sur la ponte: nombre, qualité des œufs et précocité ; sur la croissance : poids au triage et poids adulte.

Bien entendu, le troupeau a subi deux fois l'hémo-agglutination et tout porteur de germes a été éliminé.

IV. - I)'autre part, l'étude de plusieurs corrélations nous a montré l'inutilité de multiplier certaines mesures.

C'est ainsi que l'éclosivité sera exprimée en nés viables/œufs incubés, la mortalité calculée à trois semaines, le poids évalué à huit semaines et à l'âge adulte.

Cette simplification comptable a permis d'ajouter pour $195^{2}$ d'autres critères de sélection, tels que l'emplumement, le sex-ratio, si possible l'angle de poitrine, enfin la notation de toute maladie.

Enfin, la nécessité d'évaluer sur une famille entière des valeurs telles que la couvaison ou la persistance, nous a fait rejeter tout triage de poulettes - et au contraire, prévoir leur étude le plus longtemps possible —, mais simplement une élimination de coquelets d'après les performances enregistrées à l'âge de trois mois.

\section{II. - ORGANISATION GENERAL DE LA SÉLECTION}

Ayant ainsi fait l'inventaire de nos souches et souligné la nécessité de les améliorer, nous avons organisé notre travail dans le sens d'une sélection génotypique rapide et efficace. Nous voici donc arrivés au stade où ayant choisi nos reproducteurs, ayant dressé le planning d'incubation et critiqué nos premières études, il nous faut établir les principes généraux qui nous guideront. Fin fait, tout le problème se ramène à l'étude de la ségrégation ou, sous une 
autre forme, à l'évaluation et à la comparaison des performances économiques d'une centaine de familles.

\section{Le choix des critères de sélection}

Il va sans dire que nous ne pouvons pas sélectionner pour cinquante caractères. Nous rechercherons les plus révélateurs, en même temps que les plus simples:

a) L'emplumement. Nous en avons écrit précéđemment. Ce caractère est noté + zéro ou -, à un jour et à huit semaines, âges auxquels les différences sont les plus visibles.

b) Poids à huit semaines. Pour simplifier les calculs, les poids sont exprimés en décagrammes. Le poids à huit semaines indique à la fois la vitesse de croissance, la précocité du sujet et l'indice de consommation. En I95I, l'étude du rapport poids à 8 semaines/indice de consommation ne faisait qu'exagérer les différences entre les poids à huit semaines. Nous avions à l'époque calculé le coefficient de corrélation.

c) Le poids adulte est pris début janvier, avant le choix définitif des reproducteurs. Cette mesure est importante. Èn effet, un sujet précoce ralentit sa croissance. C'est donc une sélection contre nature que nous effectuerons en choisissant les animaux les plus lourds adultes et à huit semaines. Le phénomène est d'ailleurs fréquent et l'on peut dire que l'instabilité de la sélection vient d'une exagération de facteurs antagonistes : ainsi poids de l'œuf et éclosivité, poids de l'œuf et nombre d'œufs, augmentation du volume des blancs et diminution du squelette, etc...

d) L'angle de poitrine est également mesuré à treize semaines. I1 permet d'évaluer le volume des blancs - d'où la valeur chair d'un sujet. Mais cette mesture nécessite l'emploi d'un compas spécial que nous ne possédons pas actuellement.

e) L'éclosivité sera exprimée en nés viables/œufs incubés. Pour une race telle que la Marans, l'étude de l'éclosivité pourra dans 1'avenir se prêter à des recherches plus précises sur la mortalité en coquille, la technique d'incubation ou même la texture de la coquille.

L'éclosivité est facilement calculée lorsque la poule est reproductrice Sinon, nous envisageons pour l'automne I 952 d'effectuer un test général d'incubation durant le mois de décembre, de façon à augmenter la probabilité de la sélection.

f) La ponte sera étudiée sous un angle général : nombre d'oufs, précocité, intensité, pauses, couvaisons, persistance, et sous un angle particulier : les œufs, poids, couleur, forme, aspect, résistance et transparence de la coquille, voire texture du blanc, couleur du jaune, etc...

g) Enfin, nous calculerons le "sex-ratio " à huit semaines, nous évaluerons la mortalité à 2 I jours et noterons toute maladie et cause de mort. 


\section{Difficultés}

Remarquons que, pour prendre ces mesures d'une façon valable, nous sommes tenus à plusieurs servitudes :

a) De façon à diminuer le plus possible l'influence du milieu (incidence $\mathrm{C}^{2}$ sur l'héritabilité), nous avons tracé le plan de la Station de façon à recevoir en un seul poulailler toutes les filles d'une même incubation, ce qui présente encore l'avantage de comparer équitablement nos races entre elles et de simplifier considérablement le travail de la main-d'œuvre. (En I95I, nous avions échelonné nos incubations par races dans un but de simplification ; en I952, la nécessité d'opérer autrement, vu la durée nécessaire de la période d'incubation pour avoir environ dix filles par mère, s'est ainsi avérée un avantage.)

Nous pensons que cette organisation est une des originalités du Centre, car la plupart des élevages de sélection généalogique séparent leurs familles en petits parquets, sans doute dans un louable dessein de clarté, mais mésestiment ainsi la valeur de la fluctuation due au milieu. En fait, ce système rend la sélection plus rigoureuse, facilite le travail et laisse la comptabilité claire du moment que les animaux sont rationnellement immatriculés.

b) Certaines mesures sont délicates à prendre. Nous avons déjà parlé de l'éclosivité. De même, la persistance. Si nous gardons une poule sur dix, la valeur de sa persistance ne sera pas une bonne estimation de celle de sa famille.

Nous garderons donc ses sœurs non-reproductrices en poulaillers colonies, ceux-là même dont nous venons d'exposer la nécessité. Mais ces poulaillers devront être libérés avant l'entrée en ponte des jeunes poulettes de l'année. Nous devrons donc les décongestionner peu à peu ou garder un volant d'un ou de deux poulaillers de façon à suivre toutes les familles jusqu'à la reprise de la ponte.

c) Nous aurons ainsi le " progeny-test " de Izo poules et le " sib-test " de I20 familles. Le troupeau I953 naîtra de quelques poules confirmées par leur descendance et de poulettes à tester, laissant un plus grand choix, soit environ ro p. Ioo de "yearling " et 90 p. roo de poulettes.

Nous avons dit précédemment comment le système de "double shift " permet d'augmenter l'effectif sans "inbreeding ". Mais ce système d'élevage diminue le nombre des descendants par coq et, par suite, la pression de sélection et la précision des moyennes. Quand nous aurons atteint l'effectif normal de la Station, nous envisageons de revenir au système du "single-shift".

d) Avant d'exposer l'organisation particulière du service de génétique, il convient de noter que la faiblesse de nos effectifs due à la carence de locaux nous fait encourir des risques de sélection peu efficiente ou de consanguinité, vu le choix insuffisant parmi des souches de valeur très moyenne.

Nous serons donc amenés à augmenter l'effectif de telle race aux dépens de telle autre qui pourra entrer dans une recherche de croisements de première 
génération ou d'analyse génétique. Ces croisements seront en effet nécessaires dès que nous chercherons à exploiter des phénomènes d'hétérosis ou la détermination des caractères phénotypiques que seule la sélection génotypique permet de fixer rapidement. L'effectif réduit de telle race pourra donc nous conduire à des lignées " inbred " extrêmement utiles.

\section{III. - ORGANISATION PARTICULIÉRE DE LA SÉLECTION}

Nous avons donc un troupeau $195^{2}$, réparti en neuf séries d'incubation, dont deux séries de transition entre les deux pères, perđues pour la sélection au profit des études sur l'habitat et l'alimentation.

Chacune de ces séries jouit d'un même traitement et principalement au point de vue milieu: même date de naissance, même période de chauffage sous l'éleveuse, même moment de mise en arches, même poulailler colonie.

I. - Une difficulté à résoudre est donc le moyen de comparer entre elles ces différentes séries. Tous les ouvrages de vulgarisation s'accordent pour reconnaître que la date d'éclosion influe et retarde l'âge à l'entrée en ponte, le poids à huit semaines, etc... ROTMER a dressé des tables de correspondance, de façon qu'un individu né $2 \mathrm{I}$ jours après un autre soit défalqué d'une valeur proportionnelle dans l'estimation de ses performances.

Plus simplement, Hutr (I949) écrit que les deux descendances provenant des mêmes parents et ne différant que par le milieu, les valeurs moyennes seront considérées comme équivalentes et l'individu né huit jours plus tard sera déchargé de la différence des moyennes des detıx descendances.

Il est possible que la date d'incubation joue sur la précocité de la ponte, le poids à huit semaines, etc... non seulement par l'action du milieu, mais aussi par une sélection gamétique différente. Mais ce serait admettre une plasticité du patrimoine héréditaire dont la démonstration dépasse nos possibilités actuelles. I1 faudrait, pour cela, soumettre différentes séries à des milieux identiques et totalement artificiels, c'est-à-dire contrôlés. Les calculs et expériences de Hays (I95I) paraissent confirmer le point de vue de Hurr.

L. P. Cochez, appliquant depuis sept ans sur son troupeau cette méthode avec succès, compare les moyennes des descendances en calculant le probit de chaque individu pour chaque mesure, ce qui normalise légèrement les distributions par transformation linéaire.

Plus simplement encore, nous avons dressé le tableau de la fonction

$$
\pi(x)=\int_{-\infty}^{x} \frac{I}{\sqrt{2 \pi}} e^{\frac{-x^{2}}{2}} d x
$$

de façon à calculer les écarts à la moyenne de centième en centième d'écartréduit. Il a paru en effet plus avantageux de dresser cette table en centièmes d'écart-réduit qu'en centièmes de pourcentage, vu le groupement de ceux-ci vers la moyenne. 
Les avantages de l'écart-réduit résident dans sa moyenne nulle et la valeur unité de l'écart-type de la loi normale réduite, ce qui ramène par exemple le coefficient de corrélation $r$ à :

$$
r=\frac{\mathrm{XXY}}{\mathrm{N}-\mathrm{I}} \text {. }
$$

Pour effectuer le calcul de l'écart-réduit, il suffit de grouper les données en une douzaine de classes et de cumuler les pourcentages partiels de la distribution. La fonction $\pi(x)$ donne alors la valeur en écart-réduit des points médians, et il suffit d'interpoler entre classes à la précision de la mesure pour avoir la valeur écart-réduit des différents individus. Ainsi, le sujet moyen des séries I et 2 aura la valeur zéro. En tant que génotypes, les sujets + I du lot I auront la même valeur que les sujets + I du lot 2 . On voit comment, par cet artifice de calcul, une donnée brute : $650 \mathrm{~g}$ à huit semaines ou 80 œufs au I ${ }^{\mathrm{er}}$ janvier, peut révéler un avantage de l'individu de $620 \mathrm{~g}$ ou de 72 œufs né quinze jours plus tard : son écart-réduit est supérieur, du fait que l'influence du milieu a été soustraite du calcul (alors que dans la non-randomisation des familles, c'est la mesure elle-même qui est entachée d'erreur par l'action différentielle du milieu).

II. - Ayant ainsi tracé les grandes lignes du travail à entreprendre, nous avons composé une feuille de famille qui réunit les performances des parents et de leurs descendants et la valeur de ceux-ci en écarts-réduits. Il ne restera plus qu'à choisir pour reproducteurs les meilleurs individus des familles ayant eu les meilleurs écarts-réduits.

Un problème particulier s'est posé pour notre troupeau, qui est celui de la pluralité des races.

En effet, lorsque nous mesurerons le poids à huit semaines (le 29 avril pour la première fois), il est possible que les Marans se groupent en queue de distribution et les Faverolles en tête. Nous ne pourrons donc pas choisir d'une façon simple pour reproducteurs les individus dépassant par exemple la valeur + I,I7. D'autre part, si nous formons la distribution particulière de chaque race, l'effectif sera peut-être trop restreint (notamment en Faverolles et en Marans) pour que leur distribution soit assimilable à une loi de Gauss. Dans ce cas, nous pensons grouper toutes les données, ou mieux les classer par sexes, et sélectionner les reproducteurs des différentes races en fonction de l'écart-réduit moyen de chacune.

III. - Enfin, il convient de signaler quelques servitudes du point de vue "technique opératoire".

C'est ainsi que l'éclosion a nécessité la confection de paniers pedigrés, la préparation d'un cahier d'éclosion, d'un tableau de bagues d'un jour et que le détail de l'incubation, comme celui de la ponte, est porté sur une feuille individuelle.

A quinze jours, le développement de la membrane alaire permet la pose 
d'un cadenas permanent portant le même numéro que la bague d'un jour. En I95I, Io à I 5 p. Ioo des cadenas posés à la naissance ont été perdus. En I952, pas un seul cas n'est encore signalé. A l'entrée en ponte, ou peu avant, les poulettes seront agrémentées d'une plaque plus grande et partant plus lisible (actuellement à l'étude) de façon à ce que les filles d'une même mère portent des numéros se suivant. Il est en effet indispensable d'organiser l'immatriculation parfaite des animaux d'une façon rapide et simple (nous avons prévu, dans tous les cas, un double contrôle par deux opérateurs différents) afin de permettre une comptabilité claire et facile malgré la randomisation et l'attribution exacte de telle mesure à tel individu.

Réalisons en effet que le travail de sélection I952 représente le relevé brut d'environ 30 ooo à 40 ooo chiffres, auxquels il faut ajouter les calculs des écartsréduits, sans compter, bien entendu, le marquage des œufs au nid-trappe.

\section{CONCLUSION}

Le travail ainsi schématisé n'est qu'un début. Le principe de l'estimation statistique et la nécessité de "randomiser " les familles sont adoptés et actuellement réalis és pour les quatre mois écoulés. Mais, en plus de la réalisation totale de ce programme, il nous faut également prévoir nos objectifs futurs : augmentation de l'effectif, croisement et analyșes génétiques, taux d'héritabilité, étude de corrélations physiologiques, étude théorique et construction d'un index de production, développement des critères généraux et particuliers de la sélection (notamment résistance aux maladies).

Disons donc que ces lignes ne représentent qu'un canevas sur lequel viendront se dessiner toutes les complications auxquelles doit satisfaire un programme de travail soucieux à la fois d'opportunité, d'amélioration et d'organisation. On n'embrasse pas impunément trop d'activités et c'est pourquoi nous avons pensé qu'il valait mieux d'abord établir les méthodes de travail et les appliquer à des critères relativement simples mais efficaces, qu'embourber dès le départ dans des problèmes annexes une œuvre de création.

\section{Reçu pour publication le 27 avril 1952.}

\section{BIBLIOGRAPHIE}

Fisher (R. A.). - I 949 . The theory of inbreeding.

HutT (F. B.). - 1949. Genetics of the Fowl.

LERNER (I. M.). - I950. Population genetics and animal improvement.

MANN (G. E.). - I950. Poultry Breeding.

ASMUNDSON (V. S.) et LERNER (I. M.). - I 95I. Breeding chickens for meat production.

Lamotte (M.). - I948. Introduction à la Biologie quantitative.

Poultry Science. Années 1933 à 1952. 